\title{
MOOC en apreciación musical básica: proceso de reflexión crítica sobre su creación e implementación
}

\section{MOOC in basic musical appreciation: Critical reflection process on its creation and implementation}

\author{
Neiver Francisco Escobar Domínguez ${ }^{1}$, Miguel Francisco Crespo ${ }^{2}$ iD \\ ${ }^{1}$ Maestría en e-Learning, Universidad Autónoma de Bucaramanga, Bucaramanga, Colombia \\ ${ }^{2}$ Grupo Interdisciplinario de Investigaciones Sistémico Interpretativas S.C., Torreón, México \\ nescobar835@unab.edu.co, migcrespo@hotmail.com
}

(Recibido: 2 Noviembre 2017, aceptado: 1 Diciembre 2017)

\begin{abstract}
Resumen. El presente artículo es producto de un trabajo de investigación desarrollado en el seno de la maestría en E-Learning que ofrecen la Universidad Autónoma de Bucaramanga y la Universidad Abierta de Cataluña, cuyo producto principal fue el desarrollo de un MOOC dedicado al estudio de la apreciación musical básica, como estrategia de proyección social, y al apoyo a la presencialidad en el Conservatorio Antonio María Valencia, de la ciudad de Santiago de Cali. La reflexión aquí expuesta parte de la pregunta por la pertinencia de diseñar MOOC destinados al aprendizaje de la música, además de la necesidad de identificar algunas prácticas pedagógicas aplicables a la formación profesional en música. Como resultado del proceso reflexivo, se obtienen orientaciones generales para el diseño de cursos masivos en línea, especialmente aquellos destinados al aprendizaje teóricoconceptual de la música.
\end{abstract}

Palabras clave: MOOC; Sistematización de experiencia; Prácticas pedagógicas; Teoría musical; Educación superior.

\begin{abstract}
This article is the product of a thorough research work developed within the E-Learning Master degree offered jointly by the Autonomous University of Bucaramanga and the Open University of Cataluña, whose main product was the development of a MOOC dedicated to the study of Basic Musical Appreciation, as a social projection strategy and supporting the presence in Santiago de Cali's conservatory Antonio María Valencia. The insight exposed here, comes from asking about the relevance of designing MOOCs centered in the learning of music, as well as the need for identifying some pedagogic practices that are applicable to music professional development. As a result of the cognitive process, general guidelines for the design of Online Mainstream Courses are generated, especially those aimed to the conceptual and theoretical learning of music.
\end{abstract}

Keywords: MOOC; Systematization of experience; Pedagogical practices; Musical theory; University education.

\section{Introducción}

El presente escrito intenta presentar algunas reflexiones que condujeron a decidir sobre la forma y el contenido del curso diseñado alrededor de la construcción de un MOOC para la enseñanza de la teoría musical. Se trata, en principio, de poner en cuestión la pertinencia, en el contexto actual, de desarrollar un MOOC que cumpla con el propósito anunciado, a saber, encontrar una táctica innovadora, que integren las TIC como herramientas que invitan al aprendizaje autónomo y fortalecen las competencias, en beneficio de la generación de nuevos conocimientos. Dicha exploración estuvo orientada hacia la 
sistematización de la experiencia de una práctica pedagógica cuyo eje temático fuera la formación musical tradicional y la manera en que, de la presencialidad, se migra hacia un ambiente virtual.

Transformar las prácticas pedagógicas convencionales en otros enfoques teóricos se ha convertido en centro de atención de las instituciones de educación superior (IES). ¿Por qué? ¿Cuáles son las razones de fondo para que se persigan tales cambios? La cuestión por la pertinencia de las modificaciones de las prácticas pedagógicas conduce al planteamiento de una problemática que puede tratarse desde distintos niveles o perspectivas.

En un nivel más superficial, se puede hablar de los desafíos implicados en la construcción de la llamada sociedad del conocimiento. Como señala De Pablos [1] dicho fenómeno está vinculado de manera estrecha con la globalización y, muy especialmente, con la parte económica de la misma, pues convierte la información y el conocimiento en un factor de susceptible valor monetario, profundizando las desigualdades sociales ya existentes. Por esto, es importante cerrar las brechas educativas, en particular la digital.

Por otra parte, en un nivel más profundo de la problemática, está el diagnóstico sobre el cambio de época que vive la cultura occidental en el presente, formulado, entre otros pensadores contemporáneos, por Fuenmayor [2]. De acuerdo con él, el modelo educativo que se generalizó en el mundo surgió como expresión de una época ontológica, cuyo basamento se ha desmoronado. El gran propósito de ese modelo, así como sus formas y contenidos, era respuesta a necesidades histórico-culturales concretas que ya no existen más. De este modo, la búsqueda de nuevas prácticas pedagógicas está inscrita en un proyecto mayor, que implica la totalidad del sistema educativo.

No obstante, esa búsqueda se realiza en un escenario que se caracteriza por las amenazas crecientes contra la excelencia educativa, señaladas por Crespo [3]. Estas, aparentemente, compelen a buscar métodos de enseñanza, pero, en el fondo, aspiran a fines como el entretenimiento, o el simple lucro. No se sugiere que tales propósitos sean, en sí mismos, negativos, sino que se señala la hipertrofia con que, actualmente, suelen manifestarse en nuestra cultura. Su consecuencia principal es el olvido del sentido formativo que debería tener la educación, para poder cumplir su función principal.

Por otra parte, la formación profesional en música no puede escapar de los mencionados procesos de transformación e, incluso, ha visto en esta necesidad de cambio un gigantesco potencial, como política de proyección social y como mecanismo para el fortalecimiento académico y de los procesos inquisitivos. Es necesario resaltar que la enseñanza musical supone la preservación de una larga tradición —o tradiciones, si se quiere - en torno a un arte, que no debería verse impactada negativamente por la incorporación de las nuevas formas pedagógicas. Por eso, es de la mayor relevancia reflexionar sobre los cambios que se implementan, su necesidad y, también, el peligro que representan. Esto último, tal vez, aplique a la formación en cualquier disciplina humana.

Al no existir una técnica preconcebida, la metodología hace referencia al modelo cualitativo, donde la recolección de datos, la teoría y el análisis hacen parte fundamental de la sistematización del proceso de investigación que condujo el diseño de un MOOC. Este fue titulado "Apreciación musical básica" (componente nuclear de la formación profesional en música) y propuesto para el Conservatorio Antonio María Valencia de la ciudad de Santiago de Cali, Colombia.

\section{Los MOOC como respuesta a los problemas educativos del presente}

De acuerdo con lo planteado en la Introducción, los principales problemas del presente son los desafíos que implica la sociedad del conocimiento y el recambio "epocal" que vive la cultura occidental. Sin ánimo de ser taxativos, buscamos pistas que nos indiquen si los MOOC pueden ser considerados como una respuesta pertinente a tales problemas, en el entendido que, como cualquier otra manifestación cultural del presente, también pueden, incluso simultáneamente, profundizar esos mismos problemas.

¿Qué son los MOOC? Las siglas MOOC significan Massive Open Online Course y se suelen traducir como "cursos masivos y abiertos en línea". Como su nombre lo indica, se trata de programas educativos accesibles vía internet, que aceptan un número ilimitado de alumnos a los que se les puede, o no, solicitar algunos requisitos de inscripción, que por lo general son gratuitos, o de un costo muy bajo. Como cursos 
dirigidos a un amplio número de participantes, los MOOC comparten saberes propios de la formación académica de manera abierta [4]. Aunque estos cursos comparten algunas características que los ubican como entornos virtuales de aprendizaje, presentan algunas diferencias puntuales con los cursos de $E$ learning, diferenciándose, principalmente, por su carácter abierto, de modo que sus contenidos, recursos y acceso, se encuentran al alcance de, prácticamente, cualquier usuario.

Sin embargo, ¿son los MOOC pertinentes para la época actual? En principio, Cabero los concibe como inscritos en una sociedad desescolarizada [5], como un concepto educativo orientado a la obtención de conocimientos y a la promoción del aprendizaje autónomo y, a la vez, como un dispositivo tecnológico cuyos contenidos en línea se ponen al servicio de la comunidad. Ello apunta a una pertinencia positiva, sobre todo si nos enfocamos en los retos que implican la sociedad del conocimiento y la brecha digital, en el contexto del recambio epocal.

Al superar los límites de espacio y tiempo, se cuentan entre las grandes ventajas de los MOOC la masificación, la flexibilidad y los nuevos roles asumidos por docentes y discentes, desde nuevas posturas orientadoras, protagónicas y de trabajo colaborativo. Podría decirse, con ello, que este modelo es una necesidad más que un privilegio, pues proyecta, si se quiere, un punto de equilibrio entre el pico de expectativa y el abismo de la desilusión, que ostenta, en la actualidad, una meseta de productividad en aumento. Estos instrumentos de aprendizaje, caracterizados por tener un modelo enfocado hacia entornos sociales y colaborativos, promueven la creatividad, la autonomía y las estrategias en red, aportando significativamente a la generación de conocimiento colectivo.

Por supuesto, esas mismas características, favorables desde una cierta perspectiva, entrañan ciertos peligros que conviene tener presentes, al menos, como interrogantes que esperan ser atendidos en el futuro próximo, sin dejarlos de lado al momento de realizar el diseño. ¿Hasta dónde la masificación del conocimiento es realmente posible e, incluso, deseable? Lejos de la presencia física del docente, ¿cómo se evitan las interpretaciones equivocadas y las malas comprensiones? ¿Es válida cualquier posible lectura de "lo enseñado" en el MOOC? ¿Significan los MOOC un paso más hacia la transformación ontológica de la relación maestro-aprendiz en una simple relación cliente-proveedor? Además, ¿no reducen el conocimiento a mera información? ¿Cómo evitar que así sea? ¿Hay que evitarlo? ¿Por qué?

No es posible, en este momento, llegar a determinaciones al respecto, menos todavía si, a partir de la experiencia con MOOC concretos, no son formuladas esas preguntas. Así, por el momento, es necesario continuar con la intención de desarrollar MOOC, con los cuidados que exige la consciencia sobre los peligros anunciados. Surgen, entonces, algunos interrogantes en torno a la construcción de estos cursos masivos abiertos en línea (MOOC), ¿por dónde empezar? ¿Existen lineamientos o parámetros para la construcción de estos entornos educativos? ¿Qué herramientas elegir a la hora de la implementación? No es fácil responder a estos interrogantes, teniendo en cuenta que la mayor parte de la información disponible se reduce a plataformas (comunidades), concebidas como herramientas de apoyo a la docencia y dedicadas al desarrollo de entornos educativos que promueven la adquisición de competencias profesionales o personales. Comunidades como Miradax $^{1}$ o Codaes $^{2}$, ofertan cursos basados en instrucciones necesarias (lecciones y actividades), para el desarrollo técnico del empleo del software especializado, como requerimientos, diseño, aplicativos, entre otros. Sin embargo, no orientan al usuario en la selección de criterios, generales o particulares, útiles para cursos, que cumplan con características educativas y pedagógicas en la elección temática y el enfoque a determinada población destino.

Garduño [6], por su parte, afirma que uno de los retos para los educadores de la era digital está en la elección y empleo de un modelo pedagógico acorde a las necesidades, articulado al diseño de "objetos de aprendizaje", que presente procesos y resultados de la aplicación de los mismos, teniendo en cuenta elementos pedagógicos y didácticos aplicables en entornos virtuales de la era digital. En efecto, pedagogos como Garduño evidencian un singular interés en la integración pedagogía-didáctica y la definen como un proceso adaptable al contexto actual de la realidad de un estudiante, que trasciende a posturas más activas en la construcción del conocimiento. Se relega la pasividad de la instrucción, y se considera la internet como un área de aprendizaje y no como un simple espacio de interacción cotidiano.

\footnotetext{
1 https://miriadax.net/home

2 https://www.codaes.mx/inicio.htm
} 
Por otra parte, se destaca la estrecha relación entre los MOOC y los objetos de aprendizaje (OA). Estos se definen por el Plan Ceibal [7] como una nueva manera de organizar los contenidos por componentes o módulos, los cuales conservan, entre otras, características de ajuste composicional ${ }^{3}$, autocontención, usabilidad y reutilización. No obstante, al mencionar los objetos de aprendizaje es también necesario hacer referencia a la web social, tendencia a compartir información de utilidad, sobre la cual han surgido variedad de iniciativas (OA), como wikis o blogs. Se consolida, así, lo teorizado por Siemens [8], como evidencia de la evolución de los modelos educativos en abierto.

Sin embargo, la mayoría de los estudios sobre el tema se centran en la realización de una bitácora que explique cómo se crean estos objetos, determinando componentes tecnológicos y etapas de construcción, sin mayor énfasis en su aspecto, ni en los elementos creativos y pedagógicos que se deben tener en cuenta en su configuración. Si bien Smith [9] define una estructura de creación que conserve un orden lógico de los recursos, un objetivo educativo con unas metódicas claras y mesurables es indispensable para evitar que los OA dejen de ser una simple colección de información.

Ahora bien, ya se ha definido una serie de necesidades a partir de diferentes comunidades académicas: la integración entre herramientas, características técnicas, área del conocimiento y la reutilización de los recursos. Del mismo modo, se describen los obstáculos que han interferido de alguna forma: el impacto poco progresivo de los $\mathrm{OA}$ en abierto en la Universidad, los enfoques disímiles o lenta actualización de usabilidad, reutilización y sostenibilidad, el desarrollo de las comunidades de aprendizaje y la incipiente cultura colaborativa entre los usuarios [10]. Ubicar criterios generales y específicos en la elaboración de estos objetos merece una particular atención, en busca de la integración de la formación académica, articulada con formación abierta, como característica principal de los MOOC.

Zapata [11], en Aguaded y Medina [12], destaca los MOOC como una realidad que responde a muchas necesidades de la sociedad emergente, en tanto recurso en abierto y acceso al conocimiento a través de la tecnología. Esta discusión teórica, en relación a los criterios de calidad y gestión de los MOOC, los divide entre aquellos sustentados por la base conectivista y aquellos otros centrados en el contenido. Asimismo, resalta la alta tasa de abandono (75\% al $95 \%$ ), explicada, al parecer, por la falta de calidad de los cursos, o por la adaptación de cursos presenciales con finalidades de mercadeo.

En la búsqueda de significados, inherente al proceso educativo, es importante resaltar el establecimiento de criterios de calidad específicos según los contextos académicos, en estrecha relación con la población destino. Ubicando la problemática, se identifica la herramienta y se expone gran variedad de opciones, que serán valoradas según el beneficio o la dificultad de la problemática misma, o por el contexto de sus participantes.

\section{La enseñanza musical de cara a los desafíos del presente}

\subsection{La música y la sociedad del conocimiento}

Aunque no resulta fácil definir la sociedad del conocimiento, Mateo [13] reduce la expresión a los parámetros que condicionan el saber en la sociedad actual, determinando así el bienestar y progreso de los pueblos. Este tipo de sociedad, que emergiera con las nuevas tecnologías, trata de revelar la relación directamente proporcional entre el conocimiento y la calidad de vida, asumiendo que el conocimiento se transforma en procesos o productos que terminan influenciando la estructura organizacional de la nueva sociedad. Como señala De Pablos [1], la aparición del concepto "sociedad del conocimiento" se da en el contexto de la globalización. Su uso generalizado puede entrañar una excusa para la profundización de las desigualdades en el mundo, pues ejerce una presión que fortalece una concepción homogenizante de la humanidad [14], en detrimento de la diversidad cultural. El sentimiento de arraigo y de nacionalidad se

\footnotetext{
3 Pequeñas unidades ajustables entre sí.
} 
debilita en el seno de sociedades que aspiran a "no ser dejadas de lado", por no haberse sumado a la "aldea global".

La música, por su lado, hace parte de un patrinomio cultural que, aunque en diversos momentos de la historia ha tratado de jerarquizarse, resulta siendo prácticamente indivisible: danza, literatura y música. Así pues, estas manifestaciones forman parte importante de la identidad de un pueblo, interpretaciones de una sociedad en transformación, que a la vez intenta conservar sus tradiciones. La música, como las demás manifestaciones culturales que pueden ser señaladas como signos de identidad de una sociedad en particular, en el seno de la globalización y la sociedad del conocimiento, aparecen como dispositivos para la resistencia, que, sin abandonar el afán por incorporarse al resto del mundo, permiten seguir cultivando la sensación de que "se pertenece a un lugar" y que este es, parcialmente, distinto a los otros sitios.

La música y la sociedad del conocimiento debieran tener una estrecha relación, donde se incluyan las transformaciones artístico-culturales y los aportes que afectan, de una u otra forma, a la institución social, como aportes conceptuales, críticos, reflexivos, entre otros de componente formativo. No obstante, como acertadamente menciona Campero [15], cuando aduce la industria del entretenimiento como motor fundamental de crecimiento en este sector, se trata de una revolución, que poco a poco se desliga de los patrones tradicionales para ocupar competitivamente un nuevo rol social, una oportunidad de negocio.

Estamos ante desafíos que no son técnicos, ni individuales, ni referidos a estrategias mercantilistas distantes a la orientación de criterios educativos y pedagógicos. Incluso, surgen interrogantes que ponen en tela de juicio la necesidad de la enseñanza masiva de la teoría musical, teniendo en cuenta estos conflictos interculturales. Sin embargo, apelamos a la convicción de que el arraigo es un elemento fundamental de la existencia humana. La condición humana se funda en el sentido y, para que éste exista, es necesaria la conciencia en torno a un punto de origen y de pertenencia. La posibilidad de distinguirnos de los otros, por demás necesaria en términos de nuestra propia identificación, exige saber de dónde venimos. Conocer sobre teoría musical, si bien implica una lectura no autóctona de nuestras expresiones artísticas, sienta las bases para que, desde esa misma lectura, podamos apreciar la música de otros orígenes, permitiéndonos valorar, en su justa dimensión, aquello que nos es más propio.

\subsection{MOOC para la enseñanza de la música como respuesta a los desafíos del presente}

En retrospectiva, los MOOC surgen de la necesidad de un cambio en la formación abierta mediada por la tecnología, en busca de masificación y gratuidad, a través de entornos colaborativos. Sin embargo, también enfrentan desafíos en la llamada brecha digital y en el modelo educativo de la cultura occidental, intentaremos abarcar los más relevantes. La formación profesional en música supone una larga tradición instructiva, que ha desembocado en una etapa de reflexión sobre los cambios a implementar tras el fortalecimiento de la academia, sin descuidar el enorme potencial que se ve en la proyección social (ver Figura 1). 


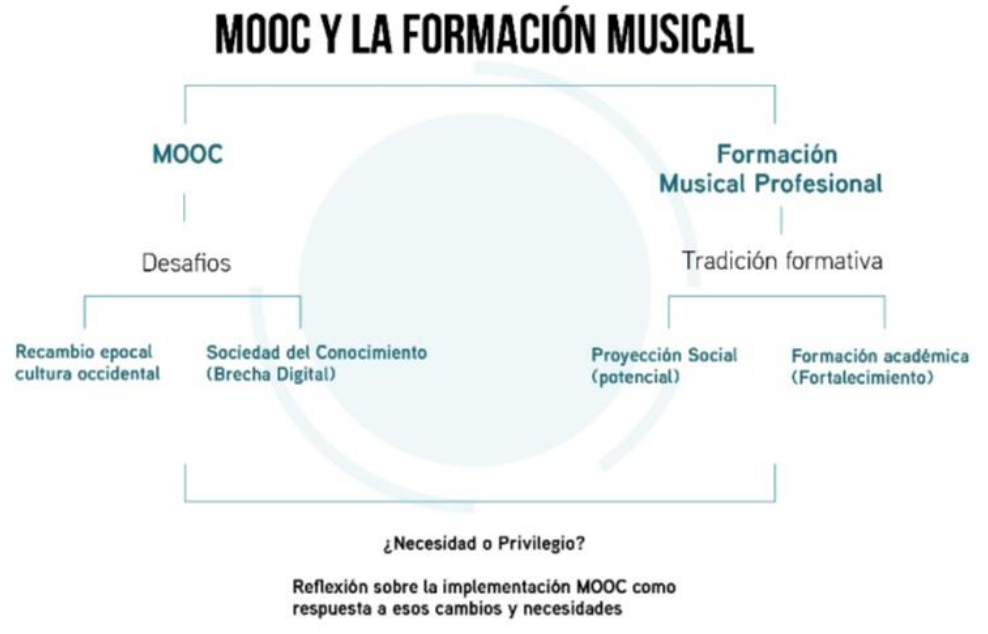

Figura 1. MOOC y la formación musical.

Ante las problemáticas de la formación musical profesional, los MOOC parecen tener la respuesta. Esta reflexión es importante para ajustar la herramienta con la que pretendemos enfrentar estos retos. ¿Contribuyen los MOOC con el cambio esperado por la academia musical? Aguaded y Medina [12] insisten en que las discusiones académicas descubren las necesidades y, asimismo, establecen las guías que marcarán el camino a seguir en el proceso de la oferta formativa. De este modo, se identifica la demanda, luego es brindada al usuario, que podrá aceptarla o no de acuerdo con sus expectativas y preferencias. En respuesta a lo anterior, se debe considerar, incluso, la pertinencia de la enseñanza masiva de teoría musical como un punto de partida o una temática amable, que se presta a la organización de contenidos en red y que contribuye a la formación de una sociedad educada musicalmente por el comercio y las tendencias del momento, marcadas por las redes sociales y los diversos medios de comunicación.

En consecuencia, los MOOC habrían superado la primera barrera de oferta y contexto y pueden ser vistos como servicios comunitarios, debido a sus ya mencionadas particularidades. No obstante, la tarea de diseño sugiere una orientación hacia el aprendizaje autónomo y a la obtención del conocimiento. Tal como lo señala Cabero [5], teniendo en cuenta sus tres grandes virtudes: masificación, flexibilidad y los nuevos roles docente-discente.

Esta etapa es fundamental para evitar reducir el conocimiento a mera información colgada en una plataforma. Aparecen, entonces, las estrategias en red en favor del conocimiento colectivo y uso de elementos creativos en la construcción de objetos de aprendizaje, que minimicen falsas interpretaciones en ausencia de un docente, como ocurre en la presencialidad, tradicional en la formación musical. En este punto, es importante que la información sea idónea y acertada, a fin de evitar insatisfacción e incredulidad hacia la institución que la promueve.

\subsection{MOOC, entre el diseño del MOOC y la teoría musical}

Cuando hablamos del carácter abierto de los MOOC, no solo hacemos referencia a la gratuidad: los debates, recursos, actividades y contenidos en general están disponibles para cualquier usuario. No obstante, la planificación debe ser aún más cuidadosa, considerando el manejo que debe darse a las licencias para contenidos digitales ${ }^{4}$.

Hacen parte efectiva de una planeación y diseño de un MOOC la elección de la plataforma, la configuración de los mecanismos de comunicación, la asignatura y la temática, el contenido y la elección

\footnotetext{
${ }^{4}$ Copyright y Copyleft.
} 
del equipo docente y técnico, el diseño de módulos, objetos de aprendizaje y la difusión. De modo similar, se elige el tipo de evaluación, que estará sujeto al modelo ${ }^{5}$, mediante test ofertados por la plataforma misma, o por actividades de entrega. Todo con sus respectivas rúbricas, basadas en competencias y criterios generales (UTEID) [16].

Los procesos de planificación, diseño e impartición de cursos MOOC para la teoría musical ${ }^{6}$ entendida en el campo de la musicología - no difieren mucho de los planteados en cualquier disciplina. Las diferencias radican en la elección de una temática específica y en la configuración de los contenidos, además de un equipo interdisciplinario que ayude en la construcción del espacio de formación (ver Tabla 1).

Tabla 1. Planificación de un MOOC en música.

\begin{tabular}{l|l}
\hline Unidad - Componente & Elementos de interés \\
\hline Plataforma del entorno & $\begin{array}{l}\text { Se elige según las necesidades del marco } \\
\text { educativo y la disponibilidad administrativa. }\end{array}$ \\
\hline Asignatura a impartir & $\begin{array}{l}\text { Elección de un área del conocimiento y de una } \\
\text { asignatura existente en la presencialidad, teniendo } \\
\text { en cuenta intereses institucionales y de su público } \\
\text { objetivo. }\end{array}$ \\
\hline Contenidos & $\begin{array}{l}\text { Vídeos, audios, infografías, etc. Materiales de } \\
\text { dominio público multimedia y texto (definen } \\
\text { diseño y equipo de trabajo). }\end{array}$ \\
\hline Equipo docente y técnico & $\begin{array}{l}\text { Docente en el área específica (contenidos) y en } \\
\text { diseño del entorno (técnico). Profesionales en } \\
\text { diferentes roles } \\
\text { administración). }\end{array}$ \\
\hline Difusión y comunicación, \\
\hline Evaluación & $\begin{array}{l}\text { Difusión institucional anclada a redes, páginas y } \\
\text { demás medios. }\end{array}$ \\
\hline
\end{tabular}

Ahora bien, el contenido abierto y disponible para la creación y generación de espacios de aprendizaje en música es escaso, sobre todo si hablamos de la disponibilidad de textos en línea, con la información necesaria, expuesta de manera concreta. Lo anterior genera inconvenientes que van más allá del reconocimiento a la propiedad intelectual, dificultando la labor orientadora del docente.

Por otra parte, la música de referencia presenta otros desafíos en relación con los derechos conexos y los agentes cambiantes que componen el mercado de la industria musical, la cual se encuentra en constante transformación, según lo exponen Monroy et al. [17]. Algunos actores o agentes, como los esquemas de mercado, los productos y productores musicales y las diferentes versiones sobre una misma obra, generan restricciones de reproducción y enlace con las plataformas de aprendizaje. Más que dificultades, la disponibilidad abierta de material de texto y audio presenta retos en relación con el diseño estructural y curricular de estos cursos con temáticas musicológicas.

Para finalizar este apartado, Gonzáles y Farnós [18] plantean la usabilidad asociada al concepto de experiencia e interacción (accesibilidad) del usuario o audiencia objetivo, donde se establece el grado de eficiencia, eficacia y satisfacción en contextos de navegabilidad específicos. El diseño en sí mismo no es usable; por tanto, la integración de elementos visuales atractivos, claros y objetivos hace de la experiencia un elemento a considerar para usuarios con destrezas TIC limitadas. Sería prudente suponer una tendencia hacia un MOOC incluyente.

\footnotetext{
5 Conectivista o de instrucción.

${ }^{6}$ El campo de la teoría musical comprende el estudio de diversos elementos, donde la apreciación es solo uno de los elementos que se atribuyen a la musicología.
} 


\section{Experiencias en torno al diseño}

En el marco de la construcción y el diseño de un entorno virtual de aprendizaje, teniendo en cuenta las características particulares de la temática abordada, el significado de la experiencia aporta un punto de referencia autoevaluativo que brinda un soporte teórico importante en la reflexión didáctica, como atributo necesario del empoderamiento de la práctica de diseño y del mejoramiento de la calidad educativa. Ante todo, desde la perspectiva del Ministerio de Educación Nacional [19], es sustancial la promoción de la experiencia como una práctica concreta de un ámbito de formación, desarrollando un aprendizaje significativo a través del fortalecimiento de competencias que atiendan las necesidades de un contexto determinado. Lo anterior posibilita el mejoramiento continuo en los diferentes estamentos que componen la academia y genera un impacto benéfico de auto-reflexión.

En términos generales, las experiencias particulares en torno a la construcción del presente MOOC no difieren mucho de las que puedan llegar a tener en otros entornos. No obstante, sí se dieron algunos detalles que bien merecen un espacio en este documento. Si bien en la presencialidad la estructura y secuencialidad de los contenidos es de vital importancia, el hilo conductor en el aprendizaje musical requiere una disposición de jerarquía composicional en favor de la claridad de los conceptos, los cuales deberán ser transversales a la formación integral de todo músico. En ocasiones, brindar ese orden lógico y articularlo a un entorno virtual (incluyendo instrucción, texto plano, enlaces de interés, complementos audiovisuales, entre otros) genera algunos inconvenientes que requieren más que la implementación de estrategias creativas. La creación de objetos de aprendizaje autónomos, sistemáticos y estructurados, así como su articulación al Moodle, son el resultado de la concentración de la información en un solo elemento educativo, sintetizando información dispersa en varios documentos de texto y documentales, que terminan por conformar una herramienta de auto-aprendizaje, auto-contenida y reutilizable.

Por otra parte, la usabilidad, entendida como el propósito funcional, y la experiencia de usuario (satisfacción durante la navegación), son términos empleados en su mayoría por tiendas en línea, pero aplicables a nuestro entorno educativo. Núñez [20] define algunos factores fundamentales en relación a este tema, resumidos en la Tabla 2. Así pues, la usabilidad y la experiencia de usuario se evidencian a través del sistema de evaluación, haciendo indispensable la incorporación de una unidad que trate de manera efectiva este tema y que genere a su vez los insumos suficientes para retro-alimentar cada aspecto del diseño y puesta en marcha del MOOC.

Tabla 2. Usabilidad y experiencia de usuario

\begin{tabular}{l|l}
\hline Factor & Definición \\
\hline Tiempos de carga & $\begin{array}{l}\text { Aplicable a todo el contenido, evitando frustraciones, abandono y } \\
\text { rebotes. }\end{array}$ \\
\hline Diseño limpio y claro & $\begin{array}{l}\text { Colores corporativos, íconos y enlaces acertados que faciliten la } \\
\text { asimilación y navegación. Indispensable adaptar a variedad de } \\
\text { dispositivos. }\end{array}$ \\
\hline Accesibilidad & $\begin{array}{l}\text { Tecnología adaptable a diferentes usuarios, independiente de sus } \\
\text { capacidades físicas, técnicas o cognitivas. }\end{array}$ \\
\hline Coherencia & $\begin{array}{l}\text { Adaptación de los campos a la mayor cantidad de posibilidades, } \\
\text { relación lógica entre sus elementos }\end{array}$ \\
\hline Claridad & $\begin{array}{l}\text { Enfocada a la población destino, aporta información necesaria para la } \\
\text { comprensión, evitando lugar a dudas. }\end{array}$ \\
\hline Usabilidad & Diseño eficiente para el cumplimiento de los objetivos. \\
\hline Utilidad & Propósito concreto, eliminando la obviedad. \\
\hline Confianza & $\begin{array}{l}\text { Orientaciones en torno a la información necesaria por parte del } \\
\text { usuario. }\end{array}$ \\
\hline Diseño y estética & Producto deseable incide en una positiva experiencia de usuario. \\
\hline Creación de valor & Llenado de expectativas de los usuarios. \\
\hline
\end{tabular}


Por último, atendiendo a observaciones anteriormente mencionadas, se abordó la necesidad de mejorar el diseño y la interfaz de usuario, lo que implicó la creación de marca y de una línea creativa (red), en beneficio de la comunicación publicitaria, como estrategia de expectativa y de concentración al usuario dentro de un mismo concepto de diseño unificador (componente visual y conceptual). Dicha concentración reduce las distracciones, al mantener la atención del participante dentro de una misma red de servicios (ver Figura 2).

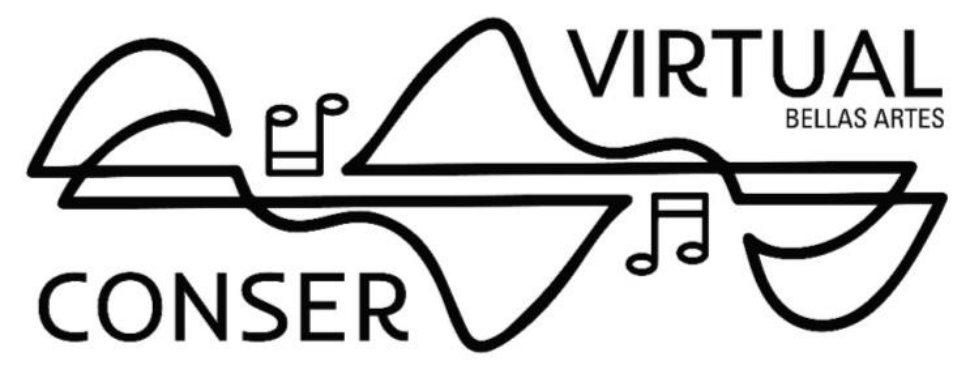

Figura 2. Conservirtual.

Avalado por diferentes instancias directivas y administrativas, Conservirtual se convierte en estandarte de la plataforma de aprendizaje del Conservatorio Antonio María Valencia, adscrito al Instituto Departamental de Bellas Artes. Este concepto de marca articula los contenidos académicos de la plataforma Moodle, con enlaces externos montados sobre canales en red, de manera tal que el usuario esté la mayor parte del tiempo vinculado a entornos bajo el mismo nombre.

\section{Consideraciones finales}

La experiencia de incursionar en el diseño e implementación de un curso de apreciación musical básica, permite reflexionar en varios aspectos. Como reflexión general, el MOOC en apreciación musical básica superó las barreras iniciales de oferta y contexto, dejando múltiples puntos de referencia para la implementación de otros cursos artísticos soportados en TIC, a partir de las características específicas de la temática abordada. Después de la experiencia vivida en torno a la prueba piloto, se vislumbran algunos aciertos y desaciertos, en concordancia con las tres grandes virtudes señaladas por Cabero [5]: masificación, flexibilidad y nuevos roles docente-discente.

La masificación se logra tras una adecuada estrategia temática y comunicativa, donde el empleo de las redes sociales jugó un importante papel. No obstante, el índice de deserción es directamente proporcional al número de participantes, para lo cual se deben implementar otras estrategias paralelas. La flexibilidad, por su parte, sigue mostrándose como la gran fortaleza de los ambientes virtuales, aunque se lucha contra la idea de algunos estudiantes, que consideran la virtualidad como sinónimo de facilidad. Por último, aunque se logró analizar el rol discente y superar algunas barreras en torno a su desempeño autónomo, el rol docente solo pudo ser analizado desde el acompañamiento de otro docente del área. Esto, aunque fue positivo, no brinda mucha información de relevancia.

La transformación de las prácticas pedagógicas tradicionales a través del MOOC rompió un poco la estructura comunicativa del relato, característica de la modalidad presencial. Sin embargo, solo presenta resultados positivos tras la creación de guías completas, claras y concisas, que no presupongan el conocimiento de ciertos conceptos. De la misma forma, el empleo de dispositivos pedagógicos que apunten a la estimulación del pensamiento reflexivo hace que la formación se centre en el estudiante, a través de una adecuada orientación, la secuencialidad del contenido y la disposición de actividades que promuevan la construcción del conocimiento. 
El diseño de un MOOC en teoría musicológica no es la solución a los problemas educativos del presente, pero sí ostenta un referente que brinda algunos elementos a considerar en la transformación de las prácticas educativas y en la implementación de más estrategias de aprendizaje soportadas en TIC. Asimismo, el consigue influenciar de manera positiva la estructura organizacional de la sociedad, desde el contexto de la globalización, y contribuir así con la responsabilidad social desde un curso de calidad, con contenidos en línea al servicio de la comunidad.

La elección de un modelo pedagógico acorde a las necesidades de una institución y una población destino, articulado a la construcción de recursos de aprendizaje autónomos y reutilizables, es un elemento clave para la evolución integral de los actuales sistemas educativos. Además, la creación de objetos de aprendizaje prioriza involucrar la mayor cantidad de sentidos en la comprensión de una percepción auditiva consciente, a falta de material consolidado, pues la información, en algunos casos, se encuentra dispersa y, en otros, no existen las suficientes fuentes digitales. Con ello, se confirma la necesidad de concentrar los contenidos en un solo dispositivo de aprendizaje. De la misma manera, articular dichos contenidos en una secuencia lógica, con la finalidad de estimular la creatividad y la intervención crítica, reflexiva y analítica por parte del estudiante, favorece los procesos de aprendizaje a través de mecanismos de participación y una metodología activa.

Las mayores dificultades se relacionaron a la disponibilidad del disperso material de consulta y a los derechos a la propiedad intelectual. Un importante acierto fue la creación de marca y la vinculación a redes sociales como mecanismo de socialización, divulgación e interacción. A través de estos canales se generó expectativa, se divulgó la existencia del curso y se publicaron contenidos. Con ello se logró que el participante estuviera más tiempo dentro de la red, lejano a elementos distractores externos. Para finalizar, conviene recordar que este estudio es solo un punto de partida para múltiples posibilidades académicas, didácticas, institucionales y personales, en torno a la investigación y creación de entornos virtuales de aprendizaje, algunos como herramienta de proyección social y otros como apoyo a la presencialidad.

\section{Referencias}

[1] J. de Pablos Pons, "Algunas reflexiones sobre las tecnologías digitales y su impacto a diferentes niveles sociales y educativos," en Comunidades virtuais de aprendizagem e identidades no ensino superior, 2007, pp. 31-38.

[2] R. Fuenmayor, El olvido del sentido holístico en la época post-moderna. 1994.

[3] M. F. Crespo Alvarado, "La importancia y el impacto de la acreditación en las IES Particulares," 2016.

[4] M. Gisbert Cervera and L. Johnson, "Educación y tecnología: nuevos escenarios de aprendizaje desde una visión transformadora," Rev. Univ. y Soc. del Conoc., vol. 12, no. 2, pp. 1-14, 2015.

[5] J. Cabero Almenara, "Visiones educativas sobre los MOOC," Rev. Iberoam. Educ. a Distancia, vol. 18, no. 2, pp. 39-60, 2015.

[6] E. Garduño Teliz, "Experiencias tecnopedagógicas en la gestión de un MOOC sobre Objetos Digitales de Aprendizaje," 2017.

[7] A. Rivoir, “"El Plan Ceibal: Impacto comunitario e inclusión social’ 2009-2010,” 2009.

[8] G. Siemens, Conociendo el conocimiento. 2006.

[9] R. S. Smith, "Guidelines for Authors of Learning Objects," 2004.

[10] Á. Fidalgo Blanco, M. L. Sein-Echaluce Lacleta, O. Borrás Gené, and F. J. García Peñalvo, "Educación en abierto: Integración de un MOOC con una asignatura académica," Teoría la Educ. Educ. y Cult. en la Soc. la Inf., vol. 15, no. 3, pp. 233-255, 2014.

[11] M. Zapata-Ros, "MOOCs, una visión crítica. El valor no está en el ejemplar," 2013.

[12] I. Aguaded and R. Medina-Salguero, "Criterios de calidad para la valoración y gestión de MOOC," Rev. Iberoam. Educ. a Distancia, vol. 18, no. 2, pp. 119-143, 2015.

[13] J. L. Mateo, "Sociedad del conocimiento," ARBOR Ciencia, Pensam. y Cult., vol. 182, no. 718, pp. 145-151, 2006.

[14] R. Fuenmayor, Sentido y sinsentido del desarrollo. 2000.

[15] H. Campero, "El negocio de la Música," 2008. [Online]. Disponible en: http://elnegociodelamusica.blogspot.com.co/2008/01/la-sociedad-del-conocimiento.html. [Consultado: 30-Jul2017].

[16] Unidad de Tecnología Educativa e Innovación Docente (UTEID), "Guía del profesor para la planificación, diseño e impartición de MOOCs". 
[17] J. C. Monroy Rodríguez, X. Rojas Murcia, J. Sáenz Ardila, and C. Arias Ospina, "El derecho de autor y los derechos conexos en la industria de la música."

[18] Á.-P. González Soto and J. D. Farnós Miró, "Usabilidad y accesibilidad para un e-learning inclusivo," Rev. Educ. Inclusiva, vol. 2, no. 1, pp. 49-60, 2009.

[19] Ministerio de Educación Nacional, “¿Qué son las experiencias significativas?," 2017. [Online]. Disponible en: http://www.colombiaaprende.edu.co/html/docentes/1596/article-197149.html. [Consultado: 30-Jul-2017].

[20] A. Nuñez, “¿Conoces la diferencia entre usabilidad y experiencia de usuario?,” 2016. [Online]. Disponible en: http://www.ticbeat.com/tecnologias/diferencia-usabilidad-experiencia-de-usuario/. [Consultado: 30-Jul-2017].

\section{Sobre los autores}

\section{Neiver Francisco Escobar Domínguez}

Licenciado en Música y Maestro en Música con línea de profundización en Musicología, egresado de la Universidad del Valle, y candidato a grado de la Maestría en E-learning de la Universidad Autónoma de Bucaramanga (UNAB), en convenio con la Universidad Abierta de Cataluña (UOC).

\section{Miguel Francisco Crespo Alvarado}

Candidatura Doctoral en Ciencias Aplicadas, Mención Sistemología Interpretativa, Universidad de los Andes, Venezuela. Maestría en Sistemología Interpretativa, Universidad de los Andes, Venezuela, 2005. Maestría en Educación, Universidad Autónoma de la Laguna, México, 2001. Maestría en Administración, Universidad Autónoma de la Laguna, 1994. Licenciatura en Administración de Recursos Humanos, Universidad Autónoma de la Laguna. 1991. Ciudad de México, México D.F. 